\title{
STUDENT ATTENDANCE SYSTEM BASED ON THE FACE RECOGNITION
}

\author{
Harshit Agarwal \\ BCA \\ School of Computing Science \\ and Engineering(SCSE) \\ Jewar, India
}

\author{
Govinda Verma \\ BCA \\ School of Computing Science \\ and Engineering(SCSE) \\ Agra, India
}

\author{
Lakshya Gupta \\ BCA \\ School of Computing Science \\ and Engineering(SCSE) \\ Meerut, India
}

\begin{abstract}
Attendance system is very important in schools and colleges' The student attendance program has many problems such as it may not be accurate and critical to maintain. Therefore, an existing system that uses a face recognition system increases accuracy and also requires less time than other methods. There are many systems available such as face recognition using IoT, PIR sensors and so on. With face recognition, hardware devices are helpful. But the challenge is to keep all the nerves properly without getting hurt. After learning all the techniques and techniques we try to use the system with Haar Cascade Algorithm with the highest accuracy among them all. It can take pictures from $50-70 \mathrm{~cm}$. We create a graphical interface that takes pictures, builds a database and trains the database with a single click. After seeing the face it will show the student's name and roll number. That information is stored on an automatic attendance sheet by time and date.
\end{abstract}

\section{INTRODUCTION}

As we have seen in many schools and colleges, members of the faculty are faced with the problem of the presence of proxies, they keep all the handwritten notes to visit each group / class every day. It is a very difficult task for them. To reduce their workload many strategies are emerging. Face recognition is the process of identifying something that has already been discovered as a known or unknown face. Often the problem of facial recognition is confusing and the problem of facial recognition Face recognition on the other hand determines whether a "face" is a well-known, or unknown, person who uses this purpose facial information to verify this facial expression.

\section{LITERATURE REVIEW-}

Attendance System based on face recognitionThe paper focused on improving school and college student attendance. Because there have been so many injustices in personal adoption as costs, illegal attendance and it may not always be so. Biometric and surface techniques are therefore recognized. The traditional methods of seeing faces were not so bad. This paper represented the idea that saving time and improving the System Attendance System using face recognition procedures was very helpful. In this sense the pictures were taken with a camera or CCTV camera at school / college with the intention of going there. He then first found the face using the features of the eyes, nose, mouth, hair and different facial expressions in the image.

\section{Automated Attendance Mgmt System Using Face Recognition \\ Introduction}

The basic concept of face recognition is image processing. There are two types of image processing: Analog Processing: It is a method used for hard copies such as photographs and printing.

Digital Performance: Controls digital photo content via PC. Basically attendance is of two types: Student Arrival Program

\section{Automatic Visiting Program.}

There are many difficulties in manual attendance such as handling all the papers and noticing the attendance / absence from the pen every day, so this takes more effort and more time. But this limitation was removed by the automated attendance system. The main purpose of the paper is to get pictures or videos of the student face, their position and appearance. There are some systems for attendance but they have some restrictions.

Biometric based system: It scans a specific part of the body like fingerprints and indicates the appearance. But even this can take time. Bluetooth system: This system is not scalable and requires up to 8 connections at once.

\section{Attendance Management Using Facial Recognition \\ Approaches for attendance till date}

Biometric Scan: The student's fingerprint is scanned and stored in the database. To register attendance, students must use a biometric method before each class.

ID Card Swipe System: The RFID chip is placed on the student ID card, which is scanned by the system and reads the chip. That card will need to be scanned in each classroom to record attendance. A 
list of current and absent students is displayed to the faculty.

\section{Student Attendance Marking Using Face Recognition in Internet of Things}

The original idea behind this solution is to improve the attendance system using face recognition technology. This reduces proxy presence and increases accuracy.

In this proposed system, facial recognition was done using arduino and sensors. The whole concept in this paper is divided into several stages-

At the initial stage the student filled in the registration form with all the details which were stored in the college database. The student's picture / image is also stored in the database. This step is required only once.

Then the classroom entrance should have a camera with sensors and microcontrollers. Here the PIR sensor is used to measure radiation from the object. It also detects the movement of objects.

The first PIR sensor measures the radiation of an object and motion as the student enters the classroom. While the radiation is human range, the camera activates and captures images.

After the student clicks on the images it compares that image to the database. If the image matches, it will update the database.

With the present. Otherwise found not in the database. Every update in the database about a specific student sent to their parents through mail or sms every day.

- This system also worked for faculty in same manner. For faculty there was another database had created. Using this system we could also maintain faculty attendance.

\section{WORKING SYSTEM}

Facial recognition is an advanced application biometric software that uses an in-depth learning algorithm to compare a live capture or digital image to a stored face print to verify personal identity. However, in-depth learning is a class of machine learning algorithms that use multiple layers to gradually extract high-level features from the raw input. For example, in image processing, the lower layers can detect the edges, while the upper layers can detect human concepts such as numbers or letters or faces.

Facial recognition is the process of identifying a human face in a scanned image; The extraction process involves obtaining the area of the face, such as distance, variation, angle and aspect ratio, to determine whether the object is human. We can use two applications -

1 - Node.js.

2- Node.js Express.
And in this system we can use also library for run a program. Library used are face-api.js, Tensorflow.js and face landmark recognition web Api is used for feteching facial detection.

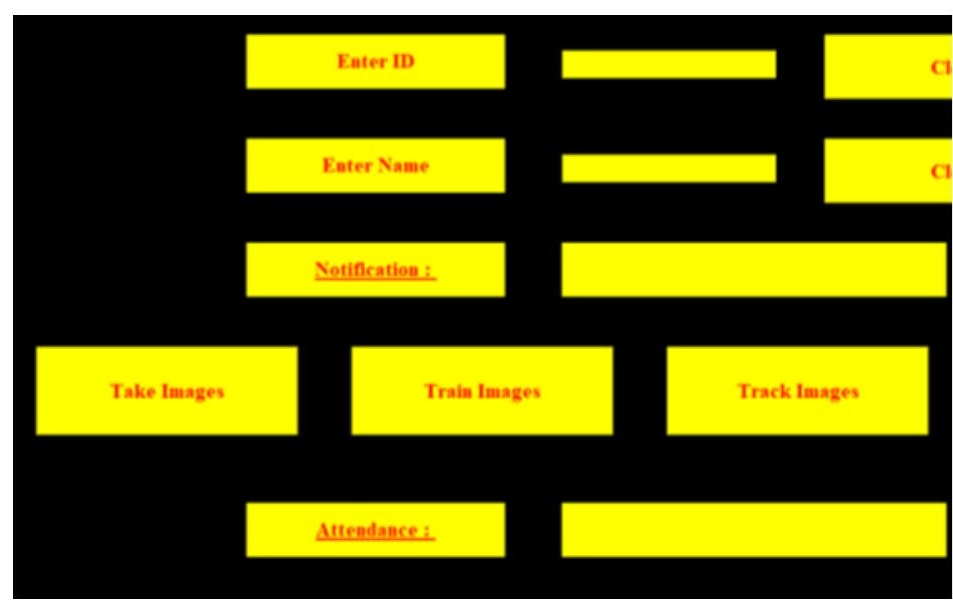

Fig 1.1 System's Dashboard

Here is our system look like, Fig1.1 shows our system dashboard in which we have to enter ID and NAME of student. After entering the ID and NAME of student we have to click on Icon named as Take Images. Then it will take image of student.

\section{Facial Recognition App using TensorFlow.js}

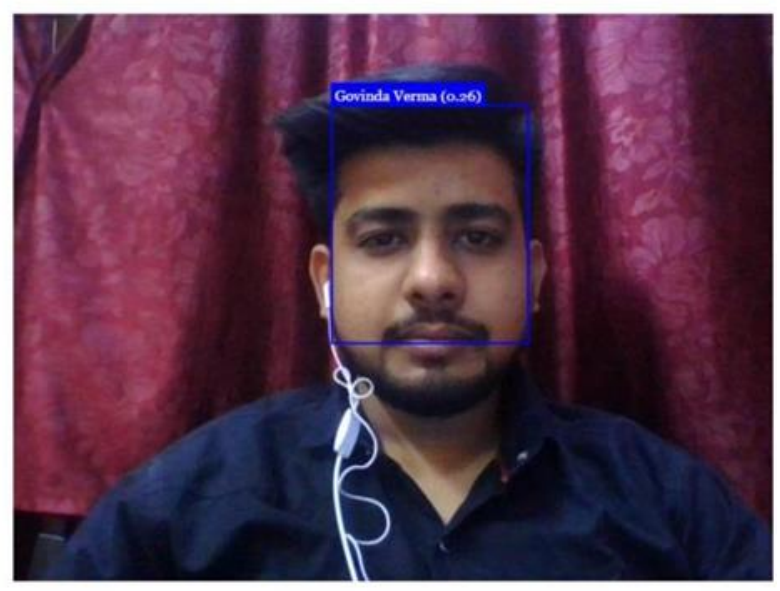

Further is that we have to track image so we have to click on Icon named as Track Image. After tracking particular image it will show the name and Id of student on his/her detected face as shown in Fig 1.2. then the information will stored in database. After completing all

At this stage we need to click on the Quit icon so that the student information is stored in the attendance as shown and it produces an excel file which is stored with the information of the students in the class. How the system works.

Let's understand the system by considering various scenarios to simplify it: 
1. How the system works if only one student enters the class:

If a student enters the classroom on time then the images of that particular student will be captured by the camera and then that image will be trained to recognize the students' faces based on the images stored in the dataset.

Based on the facial recognition of the students as shown in the information related to that particular image, an Excel file will be generated and will store the students name, roll number, date and time. The system will then mark the students as current.

A data is stored in the system the system is intended to give attendance to current students.

2. Consider the second scenario in which many students enter the classroom simultaneously. It works successfully even in that case.

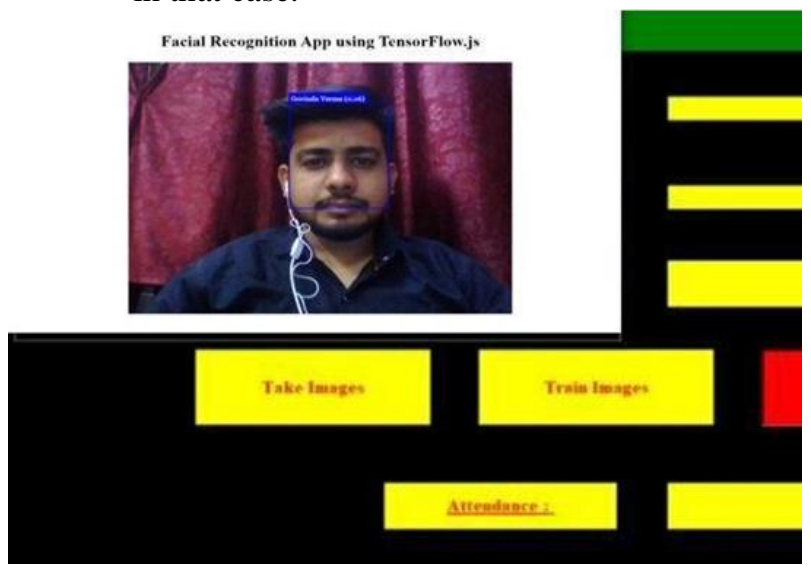

It can recognize or recognize multiple faces when entering the orbit, as shown in the picture above. After detection, when we click on the exit button it will show the presence notification in the presence box. That presence is automatically stored in the attendance sheet with ID, name, date and time.

\section{RESULT}

All information of student present in image stored in attendance sheet. So this system handles multiple faces at a time properly. Attendance sheet will update as shown in image.

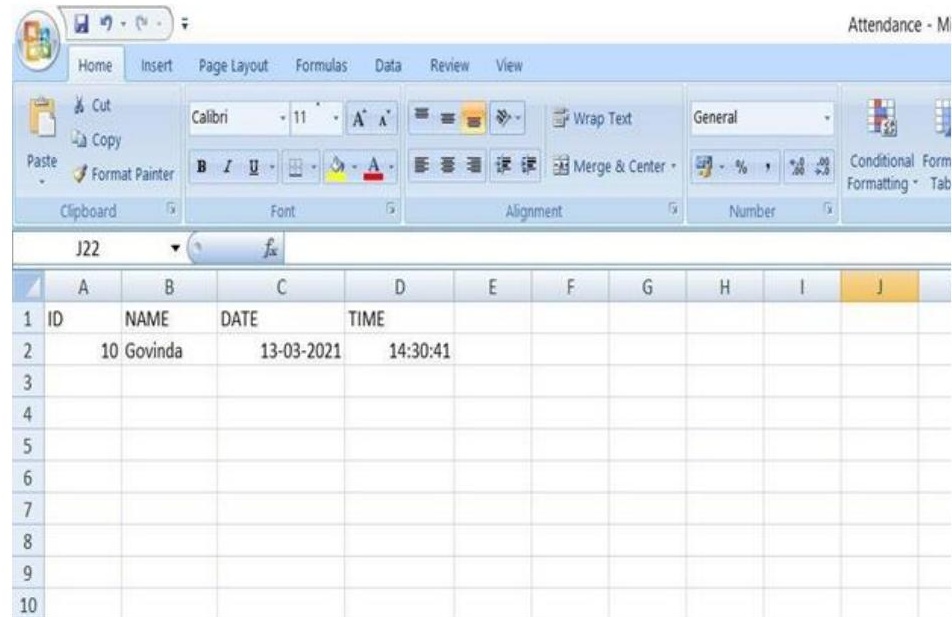

Fig. Multiple students attendances has marked

Above Fig. shows Information of student which have been stored into excel file after recognizing their faces. It stores all information of student either it is single face or multiple faces. System works properly in all conditions. When student enter into class (single/multiple) if they recognize their id and name stored in attendance sheet along with current date and time.

\section{ADVANTAGES}

1. It can handle large databases and store a large number of images for training.

2. The accuracy of this algorithm used is higher than other algorithms.

3. It is capable of capturing images very accurately from a distance of $60-80 \mathrm{~cm}$.

4. Suppose if a student accidentally enters multiple roll numbers at the time of face recognition then it shows all the roll numbers in the output.

5. Network connectivity is not required so there will be no network related problems.

6. The time it takes to create a dataset and train an image is very short.

7. Human and machine direct interface is less and which reduces many errors and increases the accuracy to a great extent.

8. The system is easy to operate during student attendance and is also easy to operate with good accuracy.

9. The system works properly for many faces and appearance updates successfully.

10. Image capturing speed is also good which takes image without getting hit.

\section{FUTURE SCOPE}

Therefore, it will be very helpful to maintain the appearance automatically with the help of facial recognition and the chances of errors will be less as compared to manual process. This will also 
reduce the rigging of attendance records of students and will also save time.

The system can be configured to detect fraud and be used in ATM machines. In addition, the system can be used at the time of use elections where the voter can be identified by recognizing the face.

\section{CONCLUSION}

In this paper, we tend to studied the use of deep neural networks on face detection and recognition. We tend to analyzed some of the different neural networks including convolutional neural networks, depth wise separable convolutions, and densely connected convolutional networks. We enforced an easy implemented a simple face detection and recognition system using Node.js and TensorFlow.js core API making use of existing API like the face-api.js package located at https://github.com/justadudewhohacks/face-

api.js/. This library is optimized to be used in the browser. Generating the data is out of the scope of this research, but there are publicly available datasets that can be used to load in the app for doing face recognition. The API allowed us to run different experiments where users can detect the emotion of a person, the gender, and the age of a person. In the last years, facial recognition has been well developed that now we can build apps that can recognize faces with great accuracy. The fascinating half concering this experiment is to seek out additional helpful application for this technology.

\section{REFFERENCES}

[1] S. Min, B. Lee, and S. Yoon, "Deep learning in bioinformatics," Briefings in Bioinformatics, 2016.

[2] G. Dahl, N. Jaitly, "Multi-task Neural Networks for QSAR Predictions," University of Toronto, 2014.

[3] K. Zhang, Z. Zhang, Z. Li and Y. Qiao, "Joint Face Detection and Alignment Using Multitask Cascaded Convolutional Networks," in IEEE Signal Processing Letters, vol. 23, no. 10, pp. 1499- 1503, Oct. 2016. doi: 10.1109/LSP.2016.2603342.

[4] S. Linnainmaa, "The representation of the cumulative rounding error of an algorithm as a Taylor expansion of the local rounding errors," Master's thesis, Univ. Helsinki, 1970.

[5] P. J. Werbos, "Beyond Regression: New Tools for Prediction and Analysis in the
Behavioral Sciences," PhD thesis, Harvard University, 1974.

[6] J. Yu and C. Li, "Face Recognition Based on Euclidean Distance and Texture Features," 2013 International Conference on Computational and Information Sciences, Shiyang, 2013, pp. 211- 213. doi: 10.1109/ICCIS.2013.63.

[7] T. Guo, J. Dong, H. Li and Y. Gao, "Simple convolutional neural network on image classification," 2017 IEEE 2nd International Conference on Big 